\title{
Long-term outcomes of patients with multivessel coronary artery disease presenting non-ST-segment elevation acute coronary syndromes
}

\author{
Piotr Desperak ${ }^{1}$, Michał Hawranek ${ }^{1}$, Paweł Gąsior ${ }^{2}$, \\ Aneta Desperak ${ }^{1}$, Andrzej Lekston ${ }^{1}$, Mariusz Gąsior ${ }^{1}$ \\ ${ }^{1} 3^{\text {rd }}$ Chair and Department of Cardiology, Medical University of Silesia in Katowice, School of Medicine \\ with the Division of Dentistry in Zabrze, Silesian Centre for Heart Diseases, Zabrze, Poland \\ ${ }^{2}$ Division of Cardiology and Structural Heart Diseases, \\ Medical University of Silesia in Katowice, Katowice, Poland
}

\begin{abstract}
Background: There is paucity of data concerning the optimal revascularization in patients with multivessel coronary artery disease (CAD) presenting non-ST-segment elevation acute coronary syndrome (NSTE-ACS). The aim was to evaluate long-term outcomes of patients with multivessel CAD presenting NSTE-ACS depending on the management after coronary angiography.

Methods: 3,166 patients with NSTE-ACS hospitalized between 2006 and 2014 were screened. After exclusions, 1,342 patients were enrolled with multivessel CAD and were divided depending on their management after coronary angiography; the medical-only therapy group $(n=91)$, the percutaneous coronary intervention (PCI) group $(n=1,122)$, the coronary artery bypass grafting $(C A B G)$ group $(n=129)$. Propensity scores matching was used to adjust for differences in patient baseline characteristics.

Results: After propensity score analysis, 273 well-matched patients were chosen. Both before and after matching, patients treated with a medical-only therapy were burdened with the highest percentage of 24-month all-cause death and non-fatal MI in comparison to PCI and CABG groups, respectively. In the CABG group, ACS-driven revascularization rate was lowest. In the overall population, PCI (HR 0.33; 95\% CI 0.20-0.53; $p<0.0001)$ and CABG (HR 0.54; 95\% CI 0.31-0.93; $p=0.028$ ) were independent factors associated with favorable 24-month prognosis. However, in a matched population only PCI was an independent predictor of long-term prognosis with a 63\% decrease of 24-month mortality (HR 0.37; 95\% CI 0.19-0.69; $p=0.0020)$.

Conclusions: In patients with multivessel CAD presenting with NSTE-ACS, medical-only management is related with adverse long-term prognosis in contrast to revascularization, which reduces 24-month mortality, especially among patients undergoing percutaneous intervention. Performance of PCI is an independent factor for improving long-term prognosis. (Cardiol J 2019; 26, 2: 157-168)

Key words: non-ST-elevation myocardial infarction, percutaneous coronary intervention, coronary bypass grafts, multivessel coronary artery disease, long-term outcomes
\end{abstract}

\section{Introduction}

Multivessel coronary artery disease (CAD) is observed in 35-70\% cases of non-ST-segment elevation acute coronary syndromes (NSTE-ACS) [1-4]. Moreover, multivessel CAD is one of the most common causes of higher risk for cardiovascular morbidity and mortality in this population [5, 6]. Although, an early invasive approach in patients with moderate-to-high risk is recommended, management of patients with confirmed multivessel $\mathrm{CAD}$ is controversial [7-9]. The guidelines suggest

Address for correspondence: Piotr Desperak, MD, $3^{\text {rd }}$ Chair and Department of Cardiology, Medical University of Silesia in Katowice, School of Medicine with the Division of Dentistry in Zabrze, Silesian Centre for Heart Diseases, ul. Skłodowskiej-Curie 9, 41-800 Zabrze, Poland, tel: +48 3237338 60, e-mail: piotr.desperak@op.pl 
that the artery responsible for ischemia should be treated first $[9,10]$. However, in the case of multivessel $\mathrm{CAD}$, subsequent treatment strategies include percutaneous coronary intervention, (PCI), coronary artery bypass grafting (CABG) or medicalonly therapy. Choice of treatment modality, completeness and optimal timing of revascularization (one- or multi-stage) of remaining lesions remains a contentious issue. Lack of detailed recommendations regarding optimal revascularization strategy is caused by a paucity of randomized trials and a small number of retrospective studies [11-14].

The main purpose of this study was to evaluate long-term outcomes of patients with multivessel CAD presenting NSTE-ACS. Therefore, an analysis was performed of clinical and angiographic status and the impact of treatment management on the incidence of 24-month all-caused death and identification of independent risk factors influencing the prognosis.

\section{Methods}

\section{Study design}

In this single-center prospective study, registry data of 3,166 consecutive patients with NSTE-ACS hospitalized from January 2006 to December 2014 were screened. Patients without invasive diagnostics during the acute phase of NSTE-ACS, with a history of CABG, with non-obstructive or single-vessel CAD were excluded from further analysis. Enrolled patients were divided into three groups depending on treatment after coronary angiography: medical-only therapy group — patients qualified for medical conservative treatment; PCI group - patients treated with PCI in the first instance; $\mathrm{CABG}$ group - patients treated $\mathrm{CABG}$ in the first instance.

The diagnosis and treatment of the study population were conducted in a highlyspecialized cardiology center with cardiac surgery facilities. Management of patients was based on current recommendations of the European Society of Cardiology (ESC) $[9,15,16]$. All patients qualified for invasive strategy have received acetylsalicylic acid and weight-adjusted unfractionated heparin. Coronary angiography was performed routinely from radial or femoral artery access depending on operator discretion. During invasive diagnostics, standard guidewires and catheters were used. After coronary angiography all decisions regarding method of treatment (medical management, PCI, CABG), in particular the use of stents, type of stent, type of cardiac surgery operation, number of grafts, periprocedural use of anticoagulants and antiplatelet drugs, and further revascularization were dependent on the decision of the operator or the Heart Team. In cases of recurrence of stenocardial symptoms associated with ST-T deviations, urgent coronary angiography was performed. Dual-antiplatelet therapy was endorsed for at least 12 months subsequent to hospitalization. Others drugs were prescribed in accordance with the ESC Guidelines [9, 15, 16]. The next stage of revascularization was routinely planned up to 3 months after index hospitalization. The adopted method of division into groups allowed the hybrid revascularization approach.

\section{Data collecting and acquisition}

Demographic, clinical and echocardiographic data regarding index hospital stay were collected by physicians and uploaded to the institutional database. Additionally, a retrospective analysis of coronary angiography, morphology and location of coronary artery lesions in all patients was conducted. 24-month follow-up data, including specific date of death, non-fatal myocardial infarction (MI) and acute coronary syndrome (ACS) driven revascularization was obtained from the official registry of the National Health Fund, guaranteeing complete data collection. Detailed data from further hospitalization planned within 3 month after discharge was also implemented to the institutional database. Follow-up data was available for whole study population.

This study was granted permission from the Institutional Review Board and University Bioethics Committee, and is in accordance with the ethical standards laid down in the 1964 Declaration of Helsinki and its later amendments [17].

\section{Definitions and endpoints}

NSTE-ACS was diagnosed on the basis of (1) clinical presentation: i) prolonged ( $>20 \mathrm{~min}$ ) anginal pain at rest, ii) new onset (de novo) angina (Class II or III of the Classification of the Canadian Cardiovascular Society), iii) recent destabilization of previously stable angina with at least Canadian Cardiovascular Society Class III angina characteristics (crescendo angina), (2) the absence of ST-segment elevation consistent with an infarction of $\geq 2 \mathrm{~mm}$ in contiguous chest leads, ST-segment elevation of $\geq 1 \mathrm{~mm}$ in 2 or more standard leads, or a new left bundle branch block and (3) after exclusion of alternative causes of chest pain $[9,15,16]$. Subsequently, patients with NSTE-ACS were classified as having unstable angina (UA) or 
non-ST-segment elevation myocardial infarction (NSTEMI) based on measured values of markers of myocardial necrosis in accordance with the Universal Definition of Myocardial Infarction [18]. Since 2009 high-sensitive cardiac troponin T was measured in the institutional central laboratory. Multivessel CAD was defined as hemodynamically significant stenosis in left main (LM) or in at least two major epicardial territories or in their major branches (left anterior descending [LAD], left circumflex or right coronary artery system) with a diameter $\geq 2.0 \mathrm{~mm}$ as determined by visual assessment with on-line quantitative coronary angiography using orthogonal views [19]. As hemodynamically significant $\geq 50 \%$ diameter stenosis in LM or proximal segment of LAD and $\geq 70 \%$ diameter stenosis in other segments were also considered. Angiographic success was defined as the achievement of a minimum stenosis diameter reduction to $<20 \%$ in the presence of TIMI flow 3 grade.

The primary outcome measure included the occurrence of 24-month all-cause death. The secondary endpoints were non-fatal recurrent myocardial infarction (MI), ACS-driven unplanned revascularization and stroke at 24 months. Nonfatal MI was defined as an ischemic event that met ESC/American College of Cardiology criteria for MI and were clearly clinically separate from the baseline ACS at the time of admission [18]. ACS-driven repeat revascularization was defined as additional, unplanned angioplasty or CABG, performed as an urgent procedure because of acute ischemic symptoms [19]. Stroke was defined as an ischemic event that was in accordance with European Stroke Organization guidelines [20].

\section{Statistical analysis}

Statistical analysis included a comparison of baseline, angiographic and procedural characteristics, and the incidence of cardiovascular events during 24-month follow-up. The analyzed variables are expressed as numbers and percentages. The distribution normality was verified using the Shapiro-Wilk test. Continuous variables were summarized using arithmetic mean with standard deviation (SD) for data following normal distribution or median with quartile 1 and 3 (Q1-Q3) for data demonstrating non-normal distribution. The analysis of variance (ANOVA) test for comparison of continuous parameters with normal distribution was performed, whereas the Kruskal-Wallis ANOVA rank test for parameters with non-normal distribution was used. Categorical variables were compared using the $\chi^{2}$ test with the Pearson's modification or with the Yates correction if the expected number of observations was less than 5 . All-cause mortality, non-fatal MI, ACS-driven revascularization and stroke in 24-month follow-up for all patients were analyzed using the KaplanMeier method with log-rank test. To minimize the confounding impact of risk factors affecting 24 -month outcomes. A propensity score analysis was performed to adjust for differences in patient baseline characteristics. First, logistic regression was performed to score all patients according to treatment (medical-only therapy vs. PCI; medicalonly therapy vs. CABG), used as covariates the clinical and procedural parameters that were clinically relevant for the endpoint: age (years), gender (male/female), diabetes mellitus, prior MI, ST-segment deviation, left ventricular ejection fraction, triple-vessel CAD and chronic total occlusion. In the next stage, analyses were performed on two matched groups (medical-only therapy vs. PCI and medical-only therapy vs. CABG), stratified into pairs to account for propensity score matching. The nearest neighbor matching was used. Both before and after propensity score matching, the Cox proportional hazards model was performed. Factors were analyzed by stepwise backward elimination ( $p<0.3$ for entry into the model, $\mathrm{p}<0.05$ to remain in the model). The independence of factors were verified by interactions testing. Results were summarized as hazard ratio (HR) with $95 \%$ confidence interval (CI). A two-sided p-value $<0.05$ was considered significant. The STATISTICA 10 software (StatSoft Inc., Tulsa, Oklahoma) was used for all calculations.

\section{Results}

During an observation period from 2006 to 2014 , a total of 3,166 patients with NSTE-ACS were analyzed (Fig. 1). After exclusions, among patients with multivessel CAD, in 91 patients medical-only treatment was implemented while in the remaining 1,251 patients revascularization was performed. Of these, 1,122 patients underwent PCI and 129 patients CABG. The average age of the study population was $66.9 \pm 10.9$ years, $68.0 \%$ were males, and the definitive diagnosis of MI was recognized in $64.2 \%$. Baseline characteristics and results of additional testing of the study groups are summarized in Table 1 . In general, the medical-only treatment group had the worst clinical profile with the highest GRACE score results. The post-hoc analysis showed that patients from 


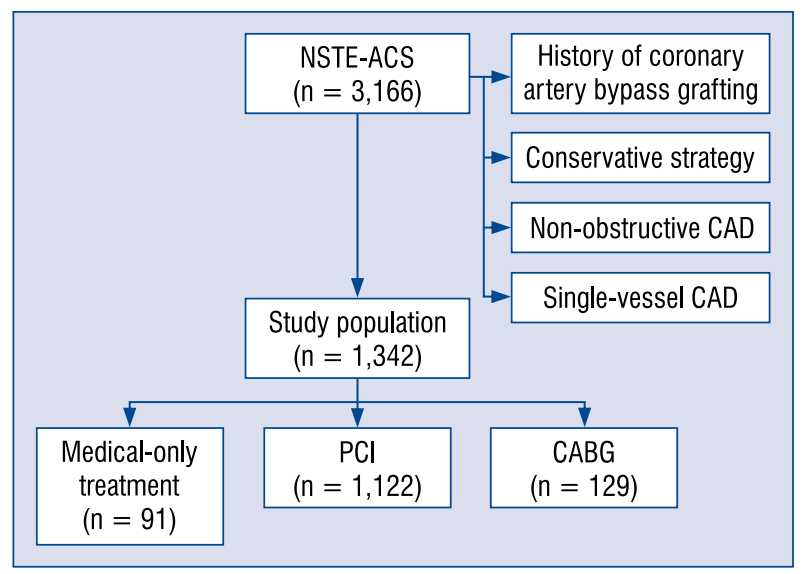

Figure 1. Study design; CABG — coronary artery bypass grafting; CAD - coronary artery disease; NSTE-ACS non-ST-segment elevation acute coronary syndromes; $\mathrm{PCl}$ - percutaneous coronary intervention.

the PCI group in comparison with $\mathrm{CABG}$ group had significantly more frequently final NSTEMI diagnosis, higher troponin $\mathrm{T}(\mathrm{p}<0.0001)$, glucose level on admission $(p=0.0029)$, lower level of left ventricular ejection fraction $(\mathrm{p}=0.0033)$, while less often arterial hypertension $(\mathrm{p}=0.012)$ and peripheral artery disease $(\mathrm{p}=0.026)$. Overall, the GRACE Risk in PCI in comparison with CABG group was higher $(\mathrm{p}=0.036)$. Angiographic and procedural characteristics are presented in Table 2. Patients qualified to medical-only treatment and to cardiac surgery demonstrated more advanced severity of coronary disease when compared to patients treated with PCI. Overall, the rate of patients undergoing hybrid revascularization was $5.7 \%$ (6.1\% in PCI group and 2.3\% in CABG group). Approximately half of patients from CABG group underwent complete anatomic revascularization after 6-month from discharge, whereas in PCI group this proportion accounted for approximately one-third of patients.

After propensity score matching of the study population group, 273 patients were selected. Patients in medical-only therapy and PCI groups had lower left ventricular ejection fraction in comparison to CABG patients. Also, the overall GRACE score was higher in medical-only therapy than in CABG group. Left main disease was more frequent in medical-only therapy and CABG than in PCI group. The other differences in baseline clinical characteristics and angiography were reduced with nonsignificant $\mathrm{p}$ value.

Table 3 contains the in-hospital, early and long-term outcomes. Kaplan-Meier curves for

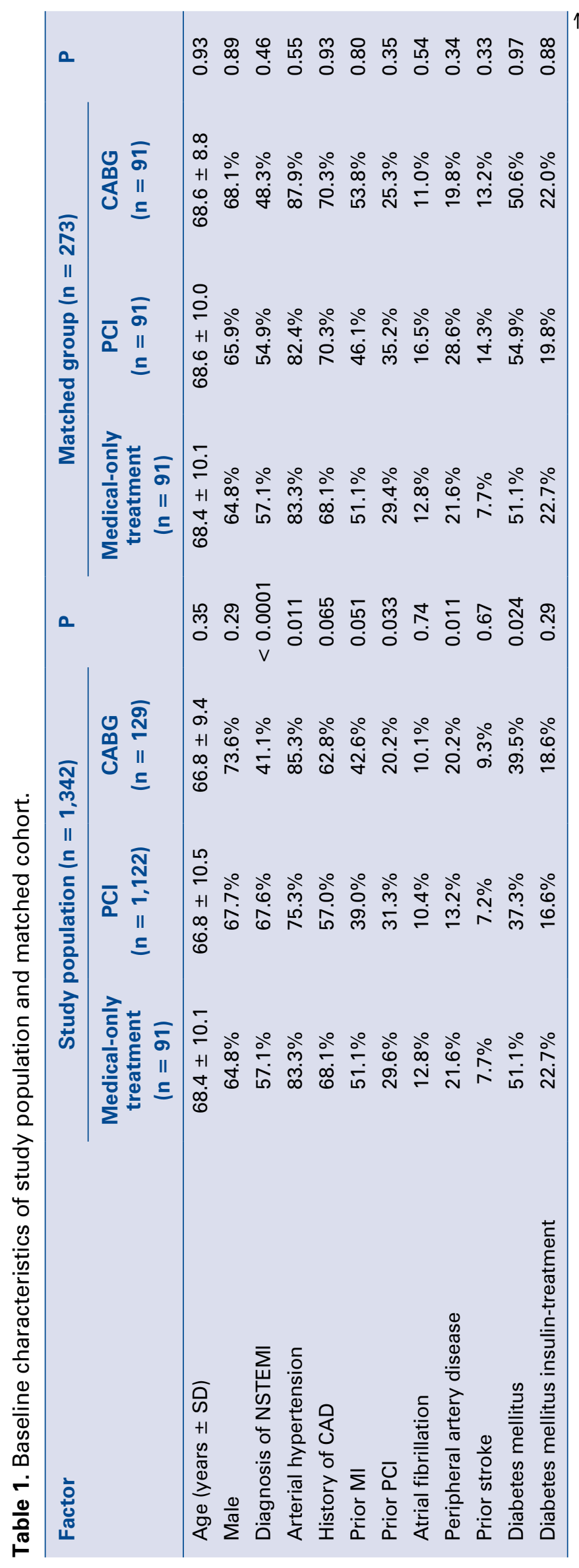




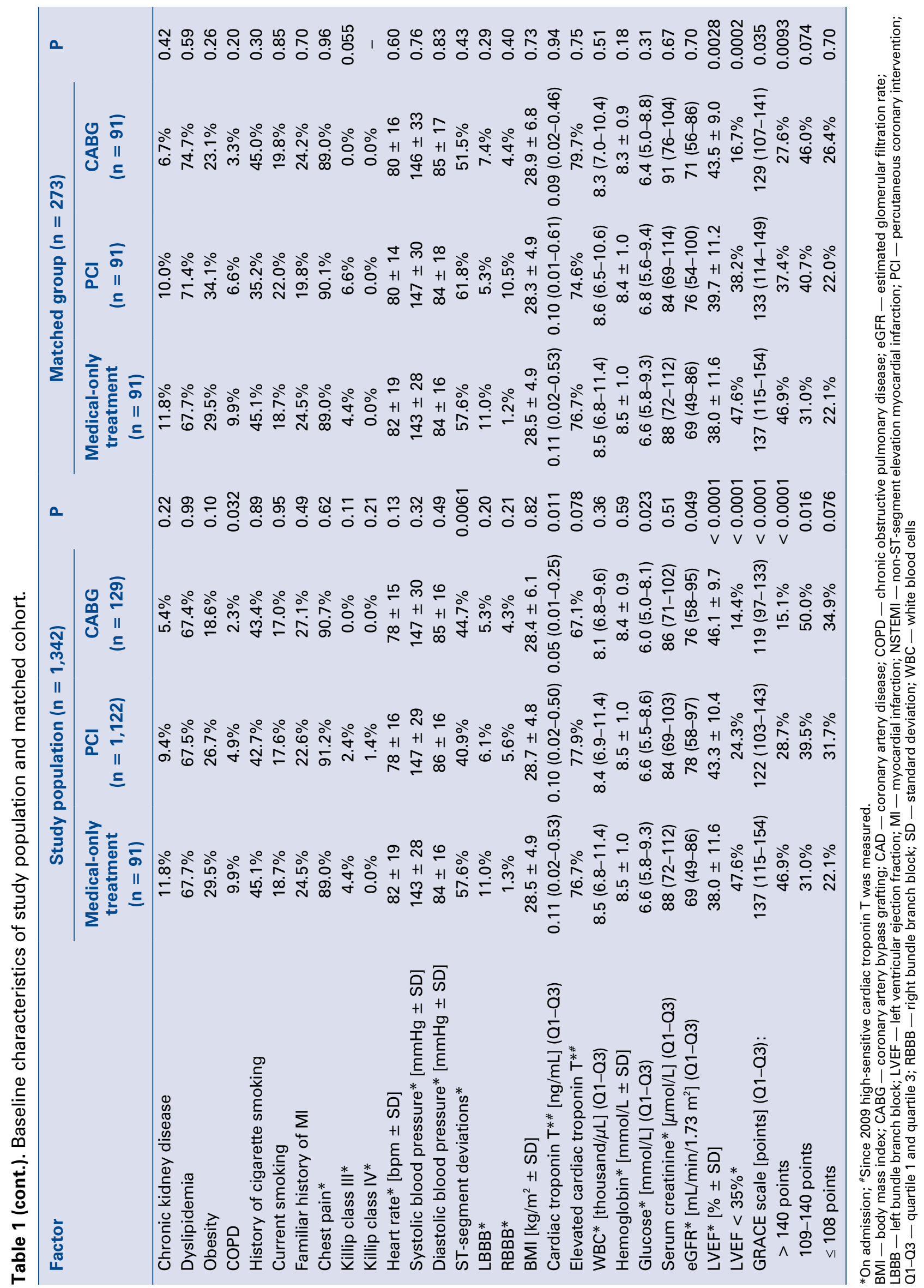




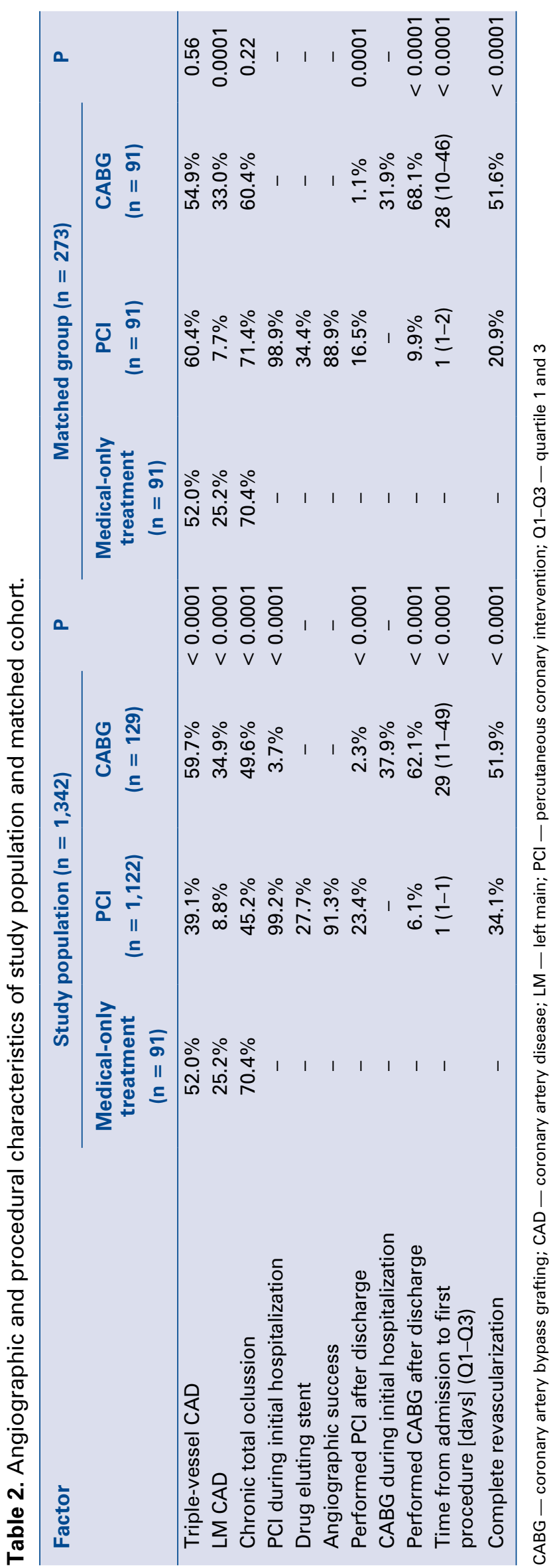

study groups are presented in Figure 2. A total percentage of 12- and 24-month all-cause death in the overall study population was $12.8 \%$ and $18.7 \%$, respectively. Patients treated with medical-only therapy were burdened with the highest percentage of 12- and 24-month all-cause death. Moreover, the highest rate of non-fatal MI in those patients was observed. In the CABG group, ACS-driven revascularization rate was the lowest. After propensity score matching, there were no differences in the incidence of 12 -month events between analyzed groups. At 24 months, the medical-only treatment was associated with the highest occurrence of all-cause death and non-fatal MI, while in PCI group had the highest rate of ACS-driven revascularization.

The Cox proportional hazards model before (A) and after (B) propensity score matching is presented in Figure 3. In the study population, PCI and $\mathrm{CABG}$ were independent factors of improved 24-month prognosis. However, in matched population only PCI was an independent predictor of 24-month prognosis with reduction of 24-month mortality by $63 \%$.

\section{Discussion}

The vast majority of contemporary studies comparing treatment strategy of multivessel CAD were performed in patients with stable angina [21-23]. Multicenter, randomized trials comparing PCI to CABG, encompassed from $13 \%$ to $91 \%$ patients with UA or recent MI [22]. In NSTE-ACS population, except for one single-center trial comparing an optimal timing of staged multivessel intervention [13], to date no prospective randomized clinical trial has been conducted to evaluate the treatment modality of multivessel CAD. Moreover, there is only a limited number of data obtained from one subanalysis of randomized trial and few retrospective studies [11, 12, 14, 24-26]. The optimal treatment method in overall as well as in particular subgroups of patients with NSTE-ACS is unclear. Therefore, the decision to conduct an assessment of treatment of multivessel CAD in real-world patients presenting with NSTE-ACS was undertaken.

In the present study comprising 3,166 patients with NSTE-ACS, the percentage of multivessel CAD without prior CABG was more than $42 \%$. The present results are similar to those previously reported for NSTE-ACS patients, where occurrence of multivessel CAD ranged from $35 \%$ to $70 \%$ [1-4]. Also, baseline clinical and angiographic characteristics of the patients appears to be 


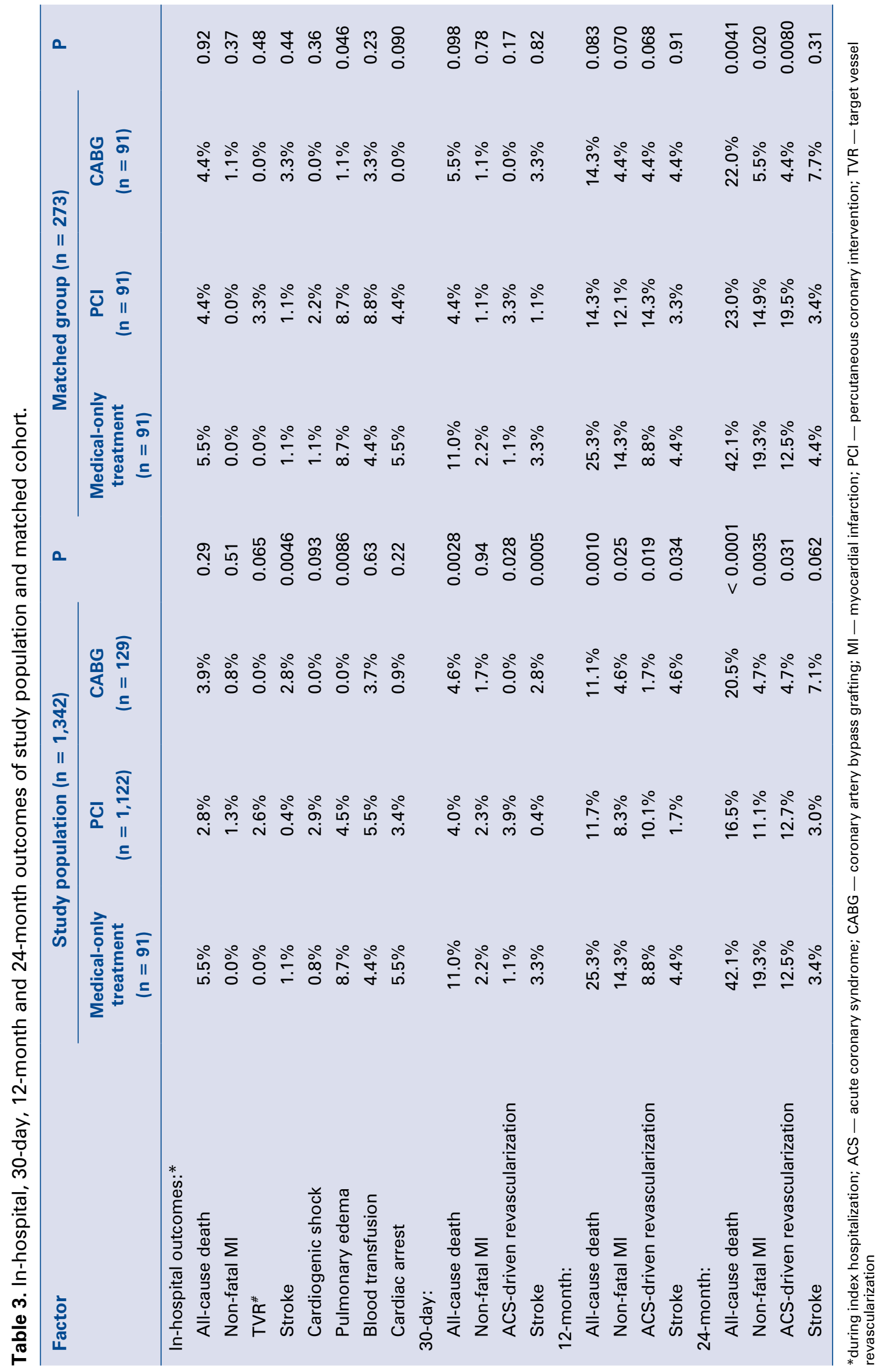



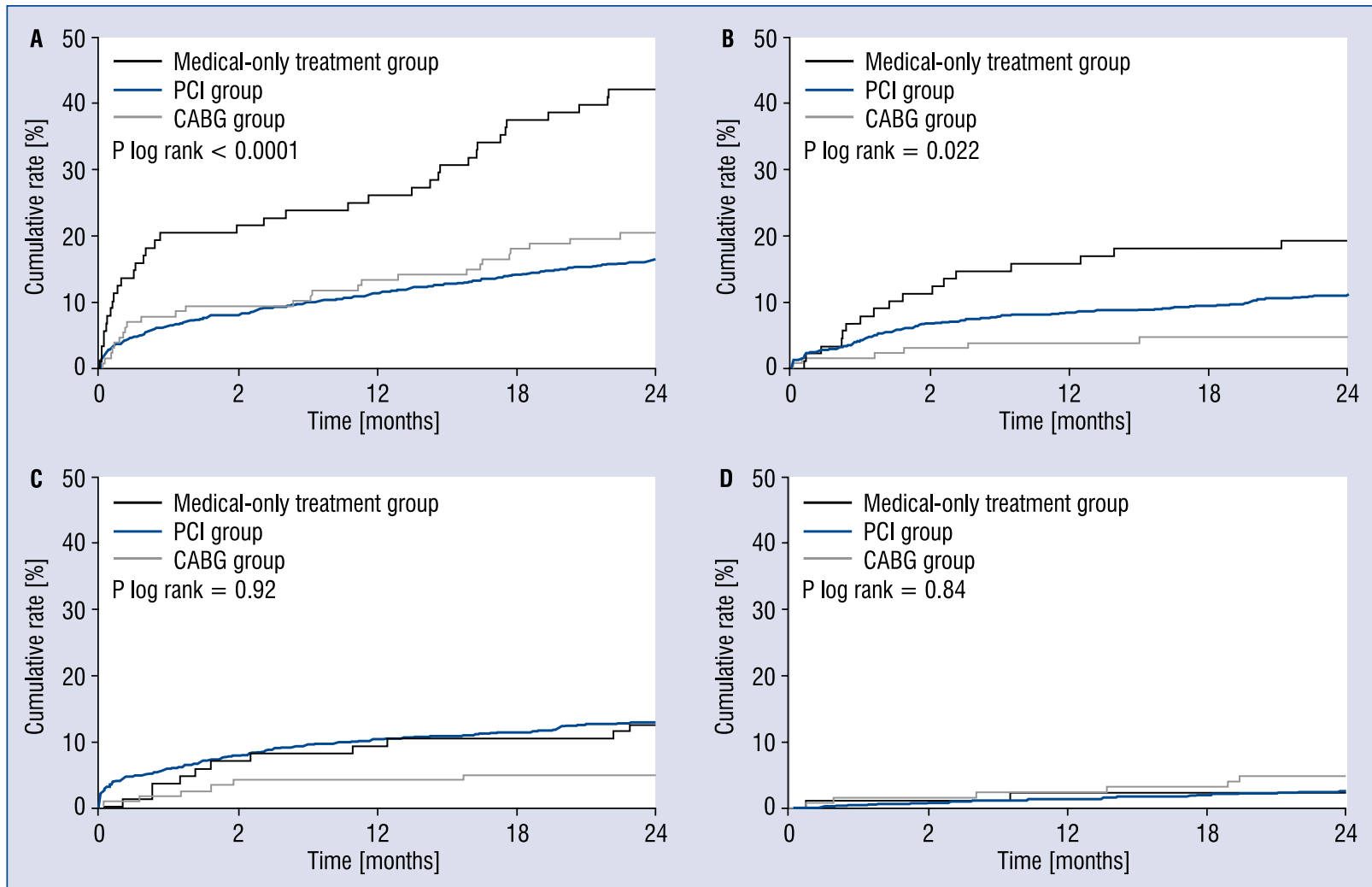

Figure 2. Kaplan-Meier survival curves for 24-month rates of all-cause death (A), non-fatal myocardial infarction (B) acute coronary syndromes-driven revascularization (C) and stroke (D) in study groups; CABG - coronary artery bypass grafting; $\mathrm{PCl}$ - percutaneous coronary intervention.

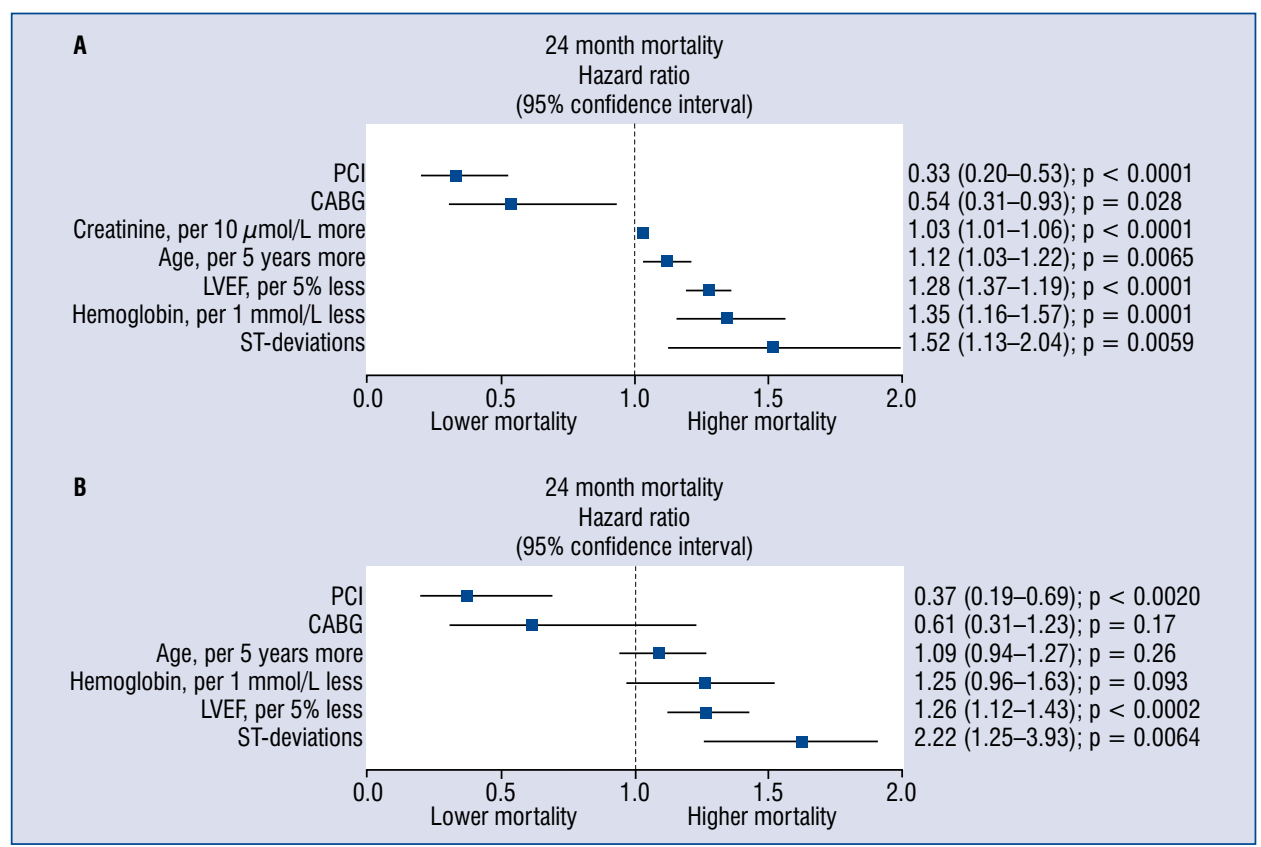

Figure 3. Forest plot of independent predictors of 24-month all-cause mortality in the study population (A) and in a matched cohort (B); CAD - coronary artery disease; $\mathrm{Cl}$ - confidence interval; LVEF — left ventrucular ejection fraction; $\mathrm{PCl}$ - percutaneous coronary intervention. 
comparable to other registries, this demonstrates a good reflection of the present patients to the general population of NSTE-ACS [11, 12, 25]. In this study, patients treated invasively constituted less than $84 \%$, surgical treatment was performed in $10 \%$ and in remaining patients the medical-only treatment was implemented. Gierlotka et al. [27] found that the rates of revascularization among Polish patients with NSTEMI shows an upward trend with contemporary use of PCI in more than $55 \%$, and $\mathrm{CABG}$ in more than $10 \%$ patients. In subanalysis of ACUITY trial, designed to compare two methods of revascularization in multivessel CAD, patients undergoing PCI accounted for $78 \%$, while CABG group consisted of $22 \%$ of study population [14]. Also, in other studies PCI was the most common method of treatment for multivessel CAD in NSTE-ACS [24-26, 28].

Early and long-term outcomes in the present analysis have shown that patients treated conservatively after coronary angiography are characterized by the worst prognosis. This result is intelligible in terms of current state of the art of management in NSTE-ACS [9, 29]. Qualification for medical-only treatment after diagnostic coronary angiography may be result of anatomical infeasibility of revascularization (i.e. rates of chronic total occlusions) and/or severe clinical status of patients [30]. The long-term outcomes were similar in PCI and CABG groups, except for higher frequency of ACS-driven revascularization in PCI group. There were no differences in the occurrence of stroke. In virtually all clinical trials, CABG was associated with higher rates of stroke in comparison with PCI [31]. The results of ACUITY trial showed that invasive treatment may be associated with lower incidence of non-fatal periprocedural MI, stroke and major bleeding, while CABG with lower occurrence of recurrent ischemia [14]. After propensity score matching analysis, early and long-term mortality in patients treated with PCI was similar to CABG group. However, an insignificant, but numerically higher incidence of the composite endpoint (25\% vs. $19 \% ; p=0.053$ ) was observed, which was mainly driven by a meaningfully higher percentage of unplanned repeat revascularization (12\% vs. $0.2 \%$; $<<0.001)$. A similar correlation demonstrating comparable efficacy of PCI and CABG in NSTE-ACS has been demonstrated in the issue of unprotected LM coronary artery [32, 33], a proximal segment of $\mathrm{LAD}[34]$ and in patients with multivessel CAD and diabetes mellitus [35]. On the other hand, in the MILESTONE Registry, immediate PCI was associated with lower long-term mortality risk compared with surgical revascularization, especially in subgroups at high clinical risk [26]. Importantly in the present study, after adjusting for factors from baseline and angiographic characteristics, the performance of PCI was an independent predictor of improved prognosis in 24-month follow-up. These meaningful outcomes are in accordance with the expert opinions that after identification a culprit vessel during coronary angiography, PCI should be the first choice procedure in the treatment of NSTE-ACS. After PCI of culprit vessel, further decisions regarding revascularization of non-ischemia-related vessels should be carried out after Heart Team consultation or based on the locally adopted proceedings protocols. PCI should also be recommended in the case of an occurrence of multiple lesions responsible for the manifestation of NSTE-ACS [36].

It is well documented that most benefits from an invasive strategy and subsequent PCI refers to patients undergoing intervention respectively in 24 (high risk) or 72 (moderate risk) hours from admission to hospital [9]. However, another important issue in multivessel CAD is optimal timing of revascularization in vessels other than the culprit vessel. There is widespread agreement of experts that in stable clinical status after intervention in the artery responsible for NSTE-ACS, treatment decisions regarding other stenosed vessels may be based on recommendations for stable CAD. In patients with severe, multivessel CAD, the preferred modality of treatment recommended by ESC is CABG. Nevertheless, outcomes of SYNTAX and EXCEL trials indicate that, the use of PCI as an alternative to cardiac surgery may be applied in patients with low-to-moderate SYNTAX score [21, $37,38]$. In the present study, more than a quarter of patients in PCI group was scheduled for the next stage of revascularization after discharge, whereas in $62 \%$ patients of CABG group, the operation was performed during further hospitalization. The results of retrospective studies and their metaanalyzes suggest that performing multivessel PCI during index hospitalization in patients presenting with NSTE-ACS may improve a long-term prognosis [11, 12]. Sardella et al. [13] in SMILE Trial has demonstrated that one-stage multivessel PCI is superior to postponed intervention. Due to a lack of randomized trials, optimal time frames of performance of CABG in NSTE-ACS patients are unclear. In accordance with expert consensus and results of clinical registries, CABG should be implemented after $48-72$ hours after performance of culprit vessel PCI, except for patients 
with ongoing myocardial ischemia, hemodynamic instability or very-high-risk coronary anatomy when there should be no delay with an operation [39]. However, the final decision should be taken by the Heart Team on the basis of clinical status and severity of CAD.

The previous data indicate that more complete revascularization of multivessel CAD may be associated with lower frequency of adverse events, particularly repeat urgent revascularization when compared to treatment limited only to the artery responsible for NSTE-ACS manifestation [11, 12 ]. Herein was found that the percentage of patients who underwent complete revascularization within 6 months after diagnosis of NSTE-ACS was more than 34\% in PCI group and more than 50\% in the CABG group. Similarly, a meta-analysis of retrospective studies proved that performance of multivessel PCI results in a reduction of long-term composite endpoint, mainly due to lower incidence of ischemia-driven revascularization. Data above indicate the need for complete revascularization in patients with multivessel CAD, if anatomical factors and the clinical condition allows.

\section{Limitations of the study}

This study was a single-center, retrospective study with potential selection biases. After coronary angiography, the further treatment decisions to perform PCI or CABG was at the operator or Heart Team discretion. The results of SYNTAX score and EuroScore were not available. Multivariate analysis may be biased because of the potential effect of confounding predictors that were not accessible in this database. A longer period of follow-up is required for more complete evaluation of PCI and CABG treatment.

\section{Conclusions}

In summary, presented results indicate that more than $90 \%$ of patients with multivessel CAD in the course of NSTE-ACS underwent coronary revascularization, of which the vast majority were treated by percutaneous intervention. The highest morbidity, risk and severe of $\mathrm{CAD}$ was observed in patients qualified for medical-only treatment. Also, medical-only management was related worse long-term prognosis in contrast to revascularization, which reduces 24 -month mortality. In addition to known factors associated with higher mortality, the use of PCI is an independent factor for improving prognosis of 24-month follow-up. Optimal revascularization method in multivessel CAD and
NSTE-ACS patients requires multicenter and randomized trials in the future.

\section{Conflict of interest: None declared}

\section{References}

1. Mehta SR, Granger CB, Boden WE, et al. TIMACS Investigators. Early versus delayed invasive intervention in acute coronary syndromes. N Engl J Med. 2009; 360(21): 2165-2175, doi: 10.1056/NEJMoa0807986, indexed in Pubmed: 19458363.

2. Thiele H, Rach J, Klein N, et al. LIPSIA-NSTEMI Trial Group. Optimal timing of invasive angiography in stable non-ST-elevation myocardial infarction: the Leipzig Immediate versus early and late PercutaneouS coronary Intervention triAl in NSTEMI (LIPSIA-NSTEMI Trial). Eur Heart J. 2012; 33(16): 2035-2043, doi: 10.1093/eurheartj/ehr418, indexed in Pubmed: 22108830.

3. Montalescot G, Bolognese L, Dudek D, et al. ACCOAST Investigators. Pretreatment with prasugrel in non-ST-segment elevation acute coronary syndromes. N Engl J Med. 2013; 369(11): 999-1010, doi: 10.1056/NEJMoa1308075, indexed in Pubmed: 23991622.

4. Halim SA, Clare RM, Newby LK, et al. Frequency, clinical and angiographic characteristics, and outcomes of high-risk non-ST-segment elevation acute coronary syndromes patients with left circumflex culprit lesions. Int J Cardiol. 2016; 203: 708-713, doi: 10.1016/j.ijcard.2015.11.036, indexed in Pubmed: 26587725.

5. Lansky AJ, Goto K, Cristea E, et al. Clinical and angiographic predictors of short- and long-term ischemic events in acute coronary syndromes: results from the Acute Catheterization and Urgent Intervention Triage strategY (ACUITY) trial. Circ Cardiovasc Interv. 2010; 3(4): 308-316, doi: 10.1161/CIRCINTERVENTIONS.109.887604, indexed in Pubmed: 20647564.

6. Beigel R, Matetzky S, Gavrielov-Yusim N, et al. ACSIS and ACSIS-PCI 2010 Investigators. Predictors of high-risk angiographic findings in patients with non-ST-segment elevation acute coronary syndrome. Catheter Cardiovasc Interv. 2014; 83(5): 677-683, doi: 10.1002/ccd.25081, indexed in Pubmed: 23784997.

7. Fox KAA, Clayton TC, Damman P, et al. FIR Collaboration. Longterm outcome of a routine versus selective invasive strategy in patients with non-ST-segment elevation acute coronary syndrome a meta-analysis of individual patient data. J Am Coll Cardiol. 2010; 55(22): 2435-2445, doi: 10.1016/j.jacc.2010.03.007, indexed in Pubmed: 20359842.

8. Katritsis DG, Siontis GCM, Kastrati A, et al. Optimal timing of coronary angiography and potential intervention in non-ST-elevation acute coronary syndromes. Eur Heart J. 2011; 32(1): 32-40, doi: 10.1093/eurheartj/ehq276, indexed in Pubmed: 20709722.

9. Roffi M, Patrono C, Collet JP, et al. Management of Acute Coronary Syndromes in Patients Presenting without Persistent ST-Segment Elevation of the European Society of Cardiology. 2015 ESC Guidelines for the management of acute coronary syndromes in patients presenting without persistent ST-segment elevation: Task Force for the Management of Acute Coronary Syndromes in Patients Presenting without Persistent ST-Segment Elevation of the European Society of Cardiology (ESC). Eur Heart J. 2016; 37(3): 267-315, doi: 10.1093/eurheartj/ehv320, indexed in Pubmed: 26320110.

10. Amsterdam EA, Wenger NK, Brindis RG, et al. American College of Cardiology, American Heart Association Task Force on 
Practice Guidelines, Society for Cardiovascular Angiography and Interventions, Society of Thoracic Surgeons, American Association for Clinical Chemistry. 2014 AHA/ACC Guideline for the Management of Patients with Non-ST-Elevation Acute Coronary Syndromes: a report of the American College of Cardiology/ /American Heart Association Task Force on Practice Guidelines. J Am Coll Cardiol. 2014; 64(24): e139-e228, doi: 10.1016/j. jacc.2014.09.017, indexed in Pubmed: 25260718.

11. Qiao Y, Li W, Mohamed S, et al. A comparison of multivessel and culprit vessel percutaneous coronary intervention in non-ST-segment elevation acute coronary syndrome patients with multivessel disease: a meta-analysis. EuroIntervention. 2015; 11(5): 525-532, doi: 10.4244/EIJV11I5A104, indexed in Pubmed: 26390516.

12. Jang JS, Jin HY, Seo JS, et al. Meta-analysis of multivessel versus culprit-only percutaneous coronary intervention in patients with non-ST-segment elevation acute coronary syndrome and multivessel coronary disease. Am J Cardiol. 2015; 115(8): 1027-1032, doi: 10.1016/j.amjcard.2015.01.530, indexed in Pubmed: 25724783.

13. Sardella G, Lucisano L, Garbo R, et al. Single-staged compared with multi-staged PCI in multivessel NSTEMI patients: The SMILE Trial. J Am Coll Cardiol. 2016; 67(3): 264-272, doi: 10.1016/j.jacc.2015.10.082, indexed in Pubmed: 26796390.

14. Ben-Gal Y, Moses J, Mehran R, et al. Surgical versus percutaneous revascularization for multivessel disease in patients with acute coronary syndromes. J Am Coll Cardiol Intv. 2010; 3(10): 1059-1067, doi: 10.1016/j.jcin.2010.06.017.

15. Hamm CW, Bassand JP, Agewall S, et al. European Society of Cardiology. ESC Guidelines for the management of acute coronary syndromes in patients presenting without persistent STsegment elevation: The Task Force for the management of acute coronary syndromes (ACS) in patients presenting without persistent ST-segment elevation of the European Society of Cardiology (ESC). Eur Heart J. 2011; 32(23): 2999-3054, doi: 10.1093/ eurheartj/ehr236, indexed in Pubmed: 21873419.

16. Bassand JP, Hamm CW, Ardissino D, et al. Guidelines for the diagnosis and treatment of non-ST-segment elevation acute coronary syndromes. Eur Heart J. 2007; 28(13): 1598-1660, doi: 10.1093/eurheartj/ehm161, indexed in Pubmed: 17569677.

17. Rickham PP. Human experimentation. Code of ethics of the world medical association. Declaration of Helsinki. Br Med J. 1964; 18: 177, indexed in Pubmed: 14150898.

18. Thygesen K, Alpert JS, White HD, et al. Joint ESC/ACCF/AHA/ WHF Task Force for the Redefinition of Myocardial Infarction. Universal definition of myocardial infarction. Eur Heart J. 2007; 28: 2525-2538, doi: 10.1093/eurheartj/ehm355.

19. Windecker S, Kohl P, Alfonso F, et al. 2014 ESC/EACTS Guidelines on myocardial revascularization: The Task Force on Myocardial Revascularization of the European Society of Cardiology (ESC) and the European Association for Cardio-Thoracic Surgery (EACTS)Developed with the special contribution of the European Association of Percutaneous Cardiovascular Interventions (EAPCI). Eur Heart J. 2014; 35(37): 2541-2619, doi: 10.1093/ eurheartj/ehu278, indexed in Pubmed: 25173339.

20. European Stroke Organisation (ESO) Executive Committee, ESO Writing Committee. Guidelines for management of ischaemic stroke and transient ischaemic attack 2008. Cerebrovasc Dis. 2008; 25(5): 457-507, doi: 10.1159/000131083, indexed in Pubmed: 18477843.
21. Mohr F, Morice MC, Kappetein A, et al. Coronary artery bypass graft surgery versus percutaneous coronary intervention in patients with three-vessel disease and left main coronary disease: 5-year follow-up of the randomised, clinical SYNTAX trial. The Lancet. 2013; 381(9867): 629-638, doi: 10.1016/s01406736(13)60141-5.

22. Hlatky MA, Boothroyd DB, Bravata DM, et al. Coronary artery bypass surgery compared with percutaneous coronary interventions for multivessel disease: a collaborative analysis of individual patient data from ten randomised trials. Lancet. 2009; 373(9670): 1190-1197, doi: 10.1016/S0140-6736(09)60552-3, indexed in Pubmed: 19303634.

23. Bravata DM, Gienger AL, McDonald KM, et al. Systematic review: the comparative effectiveness of percutaneous coronary interventions and coronary artery bypass graft surgery. Ann Intern Med. 2007; 147(10): 703-716, indexed in Pubmed: 17938385.

24. Solodky A, Behar S, Boyko V, et al. The outcome of coronary artery bypass grafting surgery among patients hospitalized with acute coronary syndrome: the Euro Heart Survey of acute coronary syndrome experience. Cardiology. 2005; 103(1): 44-47, doi: 10.1159/000081851, indexed in Pubmed: 15528900.

25. Chen LY, Lennon RJ, Grantham JA, et al. In-hospital and longterm outcomes of multivessel percutaneous coronary revascularization after acute myocardial infarction. Am J Cardiol. 2005; 95(3): 349-354, doi: 10.1016/j.amjcard.2004.09.032, indexed in Pubmed: 15670543.

26. Buszman PE, Buszman PP, Bochenek A, et al. Comparison of stenting and surgical revascularization strategy in non-ST elevation acute coronary syndromes and complex coronary artery disease (from the Milestone Registry). Am J Cardiol. 2014; 114(7): 979-987, doi: 10.1016/j.amjcard.2014.07.008, indexed in Pubmed: 25124186.

27. Gierlotka M, Gąsior M, Wilczek K, et al. Temporal trends in the treatment and outcomes of patients With non-ST-segment elevation myocardial infarction in Poland from 2004-2010 (from the Polish Registry of Acute Coronary Syndromes). Am J Cardiol. 2012; 109(6): 779-786, doi: 10.1016/j.amjcard.2011.10.041, indexed in Pubmed: 22189010.

28. de Winter RJ, Windhausen F, Cornel JH, et al. Invasive versus Conservative Treatment in Unstable Coronary Syndromes (ICTUS) Investigators. Early invasive versus selectively invasive management for acute coronary syndromes. N Engl J Med. 2005; 353(11): 1095-1104, doi: 10.1056/NEJMoa044259, indexed in Pubmed: 16162880.

29. Williams B, Menon M, Satran D, et al. Patients with coronary artery disease not amenable to traditional revascularization: prevalence and 3-year mortality. Catheter Cardiovasc Interv. 2010; 75(6): 886-891, doi: 10.1002/ccd.22431, indexed in Pubmed: 20432394.

30. Bettinger N, Palmerini T, Caixeta A, et al. Risk stratification of patients undergoing medical therapy after coronary angiography. Eur Heart J. 2016; 37(40): 3103-3110, doi: 10.1093/eurheartj/ ehv674, indexed in Pubmed: 26685136.

31. Palmerini T, Biondi-Zoccai G, Reggiani L, et al. Risk of stroke with coronary artery bypass graft surgery compared with percutaneous coronary intervention. J Am Coll Cardiol. 2012; 60(9): 798-805, doi: 10.1016/j.jacc.2011.10.912.

32. Buszman PP, Bochenek A, Konkolewska M, et al. Early and long-term outcomes after surgical and percutaneous myocardial 
revascularization in patients with non-ST-elevation acute coronary syndromes and unprotected left main disease. J Invasive Cardiol. 2009; 21(11): 564-569, indexed in Pubmed: 19901409.

33. Zhao C, Wang X, Wu X, et al. Early and long-term outcomes after percutaneous coronary intervention of unprotected left main coronary disease with drug-eluting stents in patients with non-ST-elevation acute coronary syndrome. Can J Cardiol. 2011; 27(6): 743-748, doi: 10.1016/j.cjca.2011.05.010, indexed in Pubmed: 21875777 .

34. Mennuni MG, Dangas GD, Mehran R, et al. Coronary Artery Bypass Surgery Compared With Percutaneous Coronary Intervention for Proximal Left Anterior Descending Artery Treatment in Patients With Acute Coronary Syndrome: Analysis From the ACUITY Trial. J Invasive Cardiol. 2015; 27(10): 468-473, indexed in Pubmed: 26121708.

35. Ben-Gal Y, Mohr R, Feit F, et al. Surgical versus percutaneous coronary revascularization for multivessel disease in diabetic patients with non-ST-segment-elevation acute coronary syndrome: analysis from the Acute Catheterization and Early Intervention Triage Strategy trial. Circ Cardiovasc Interv. 2015; 8(6), doi: 10.1161/CIRCINTERVENTIONS.114.002032, indexed in Pubmed: 26019142 .
36. Vergallo R, Ren X, Yonetsu T, et al. Pancoronary plaque vulnerability in patients with acute coronary syndrome and ruptured culprit plaque: a 3-vessel optical coherence tomography study. Am Heart J. 2014; 167(1): 59-67, doi: 10.1016/j.ahj.2013.10.011, indexed in Pubmed: 24332143.

37. Palmerini T, Genereux P, Caixeta A, et al. Prognostic value of the SYNTAX score in patients with acute coronary syndromes undergoing percutaneous coronary intervention: analysis from the ACUITY (Acute Catheterization and Urgent Intervention Triage StrategY) trial. J Am Coll Cardiol. 2011; 57(24): 2389-2397, doi: 10.1016/j.jacc.2011.02.032, indexed in Pubmed: 21658558.

38. Stone G, Sabik J, Serruys P, et al. Everolimus-Eluting Stents or Bypass Surgery for Left Main Coronary Artery Disease. N Engl J Med. 2016; 375(23): 2223-2235, doi: 10.1056/nejmoa1610227.

39. Parikh SV, de Lemos JA, Jessen ME, et al. CRUSADE and ACTION Registry-GWTG Participants. Timing of in-hospital coronary artery bypass graft surgery for non-ST-segment elevation myocardial infarction patients results from the National Cardiovascular Data Registry ACTION Registry-GWTG (Acute Coronary Treatment and Intervention Outcomes Network Registry-Get With The Guidelines). JACC Cardiovasc Interv. 2010; 3(4): 419-427, doi: 10.1016/j. jcin.2010.01.012, indexed in Pubmed: 20398870. 